\title{
Physics beyond the SM with kaons at NA62
}

\author{
Radoslav Marchevski*† \\ CERN
}

E-mail: Ermarchevdecern.ch

\begin{abstract}
The decay $K^{+} \rightarrow \pi^{+} v \bar{v}$, with a precisely predicted branching ratio of less than $10^{-10}$, is one of the best candidates to reveal indirect effects of new physics at the highest mass scales. The NA62 experiment at the CERN SPS is designed to measure the branching ratio of the $K^{+} \rightarrow \pi^{+} v \bar{v}$ with a decay-in-flight technique. NA62 took data so far in 2016-2018. Statistics collected in 2016 allowed NA62 to reach the Standard Model sensitivity for $K^{+} \rightarrow \pi^{+} v \bar{v}$, entering the domain of $10^{-10}$ single event sensitivity and showing the proof of principle of the experiment. Thanks to the statistics collected in 2017, NA62 surpasses the present best sensitivity. The analysis strategy is reviewed and the preliminary result from the 2017 data set is presented. The NA62 experiment at CERN collected a large sample of charged kaon decays into final states with multiple charged particles in 2016-2018. The sensitivity to a range of lepton flavour and lepton number violating kaon decays provided by this data set improves over the previously reported measurements. Results from the searches for these processes with a partial NA62 data sample are presented.
\end{abstract}

XXIX International Symposium on Lepton Photon Interactions at High Energies - LeptonPhoton2019 August 5-10, 2019

Toronto, Canada

\footnotetext{
${ }^{*}$ Speaker.

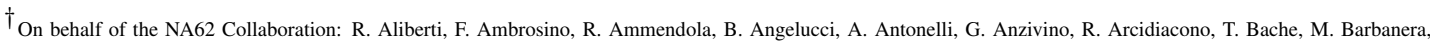
J. Bernhard, A. Biagioni, L. Bician, C. Biino, A. Bizzeti, T. Blazek, B. Bloch-Devaux, V. Bonaiuto, M. Boretto, M. Bragadireanu, D. Britton, F. Brizioli, M.B. Brunetti, D. Bryman, F. Bucci, T. Capussela, J. Carmignani, A. Ceccucci, P. Cenci, V. Cerny, C. Cerri, B. Checcucci, A. Conovaloff, P. Cooper, E. Cortina Gil, M. Corvino, F. Costantini, A. Cotta Ramusino, D. Coward, G. D’Agostini, J. Dainton, P. Dalpiaz, H. Danielsson, N. De Simone, D. Di Filippo, L. Di Lella, N. Doble, B. Dobrich, F. Duval, V. Duk, J. Engelfried, T. Enik, N. Estrada-Tristan, V. Falaleev, R. Fantechi, V. Fascianelli, L. Federici, S. Fedotov, A. Filippi, M. Fiorini, J. Fry, J. Fu, A. Fucci, L. Fulton, E. Gamberini, L. Gatignon, G. Georgiev, S. Ghinescu, A. Gianoli, M. Giorgi, S. Giudici, F. Gonnella, E. Goudzovski, C. Graham, R. Guida, E. Gushchin, F. Hahn, H. Heath, E.B. Holzer, T. Husek, O. Hutanu, D. Hutchcroft, L. Iacobuzio, E. Iacopini, E. Imbergamo, B. Jenninger, J. Jerhot, R.W. Jones, K. Kampf, V. Kekelidze, S. Kholodenko, G. Khoriauli, A. Khotyantsev, A. Kleimenova, A. Korotkova, M. Koval, V. Kozhuharov, Z. Kucerova, Y. Kudenko, J. Kunze, V. Kurochka, V. Kurshetsov, G. Lanfranchi, G. Lamanna, E. Lari, G. Latino, P. Laycock, C. Lazzeroni, M. Lenti, G. Lehmann Miotto, E. Leonardi, P. Lichard, L. Litov, R. Lollini, D. Lomidze, A. Lonardo, P. Lubrano, M. Lupi, N. Lurkin, D. Madigozhin, I. Mannelli, G. Mannocchi, A. Mapelli, F. Marchetto, R. Marchevski, S. Martellotti, P. Massarotti, K. Massri, E. Maurice, M. Medvedeva, A. Mefodev, E. Menichetti, E. Migliore, E. Minucci, M. Mirra, M. Misheva, N. Molokanova, M. Moulson, S. Movchan, M. Napolitano, I. Neri, F. Newson, A. Norton, M. Noy, T. Numao, V. Obraztsov, A. Ostankov, S. Padolski, R. Page, V. Palladino, A. Parenti, C. Parkinson, E. Pedreschi, M. Pepe, M. Perrin-Terrin, L. Peruzzo, P. Petrov, Y. Petrov, F. Petrucci, R. Piandani, M. Piccini, J. Pinzino, I. Polenkevich, L. Pontisso, Yu. Potrebenikov, D. Protopopescu, M. Raggi, A. Romano, P. Rubin, G. Ruggiero, V. Ryjov, A. Salamon, C. Santoni, G. Saracino, F. Sargeni, S. Schuchmann, V. Semenov, A. Sergi, A. Shaikhiev, S. Shkarovskiy, D. Soldi, V. Sugonyaev, M. Sozzi, T. Spadaro, F. Spinella, A. Sturgess, J. Swallow, S. Trilov, P. Valente, B. Velghe, S. Venditti, P. Vicini, R. Volpe, M. Vormstein, H. Wahl, R. Wanke, B. Wrona, O. Yushchenko, M. Zamkovsky, A. Zinchenko.
} 


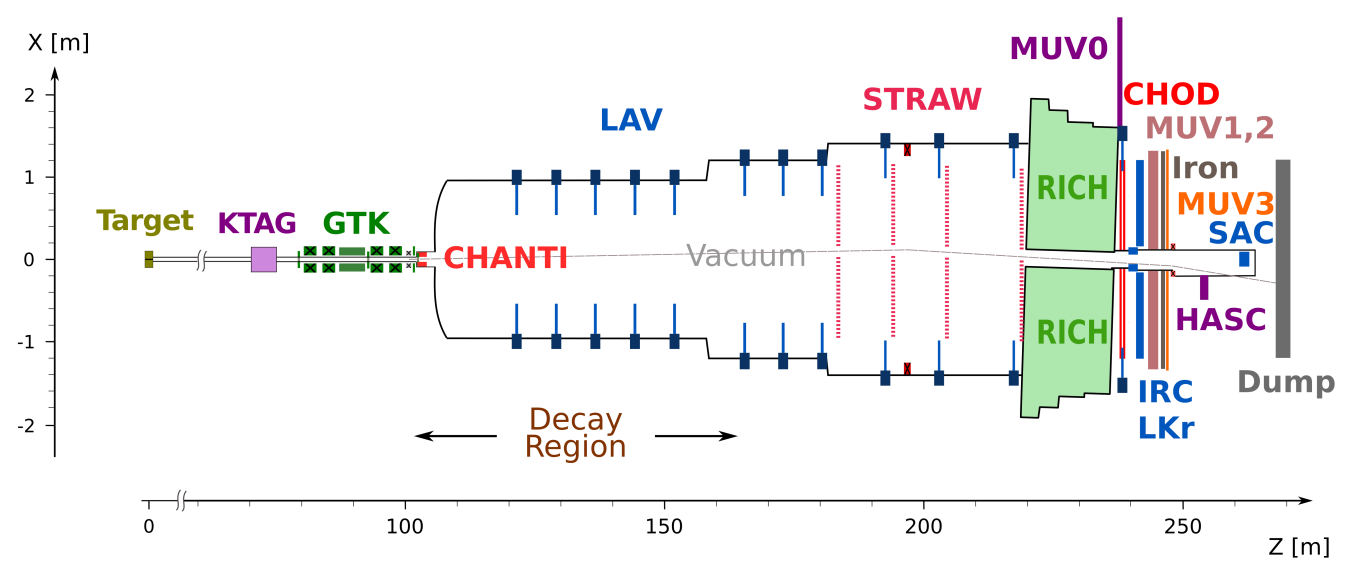

Figure 1: Schematic layout of the NA62 experiment in the $x z$ plane

\section{The $K^{+} \rightarrow \pi^{+} v \bar{v}$ decay in the $\mathrm{SM}$}

The $K^{+} \rightarrow \pi^{+} v \bar{v},\left(K_{\pi v \bar{v}}\right)$ decay is a flavour changing neutral current process proceeding through box and electroweak penguin diagrams. The process is very rare, due to quadratic GIM mechanism and strong Cabibbo suppression. The dominant contribution comes from the shortdistance physics of the top quark loop, with a small charm quark contribution and long-distance corrections. The hadronic matrix element relevant for the $K^{+}-\pi^{+}$transition can be extracted experimentally from $K^{+} \rightarrow l^{+} \pi^{0} v_{l}$ decays after correcting for isospin. This eliminates important theoretical uncertainties and makes the $K_{\pi v \bar{v}}$ decay very clean theoretically and sensitive to physics beyond the Standard Model (SM), probing the highest mass scales among the rare meson decays

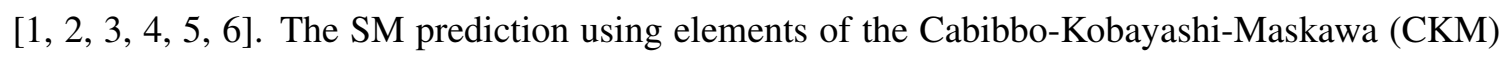
matrix extracted from tree-level processes $[\square,[]]$ is

$$
B R\left(K^{+} \rightarrow \pi v \bar{v}\right)=(8.4 \pm 1.0) \times 10^{-11} .
$$

The knowledge of the external inputs dominate the uncertainties on the predictions. The experimental result from the BNL E949 collaboration [Q]

$$
B R\left(K^{+} \rightarrow \pi v \bar{v}\right)=\left(17.3_{-10.5}^{+11.5}\right) \times 10^{-11},
$$

was obtained using stopped kaons. The branching ratio is $\sim 1 \sigma$ away from the SM prediction, but the measurement was based on only few events and the experimental uncertainties are large.

\section{The NA62 experiment}

The fixed target NA62 experiment exploits a $400 \mathrm{GeV} / c$ primary SPS proton beam. The beam impinges on a beryllium target producing secondary particles, of which the kaon component is $6 \%$. A $100 \mathrm{~m}$ long beam line selects, collimates, focuses and transports charged particles of (75.0 \pm 0.8$) \mathrm{GeV} / c$ momentum to the evacuated fiducial decay volume.

The NA62 experimental apparatus is shown in Figure $\square$. The KTAG is a differential Cherenkov detector filled with $N_{2}$ placed in the beam to identify and timestamp kaons. It is followed by the 

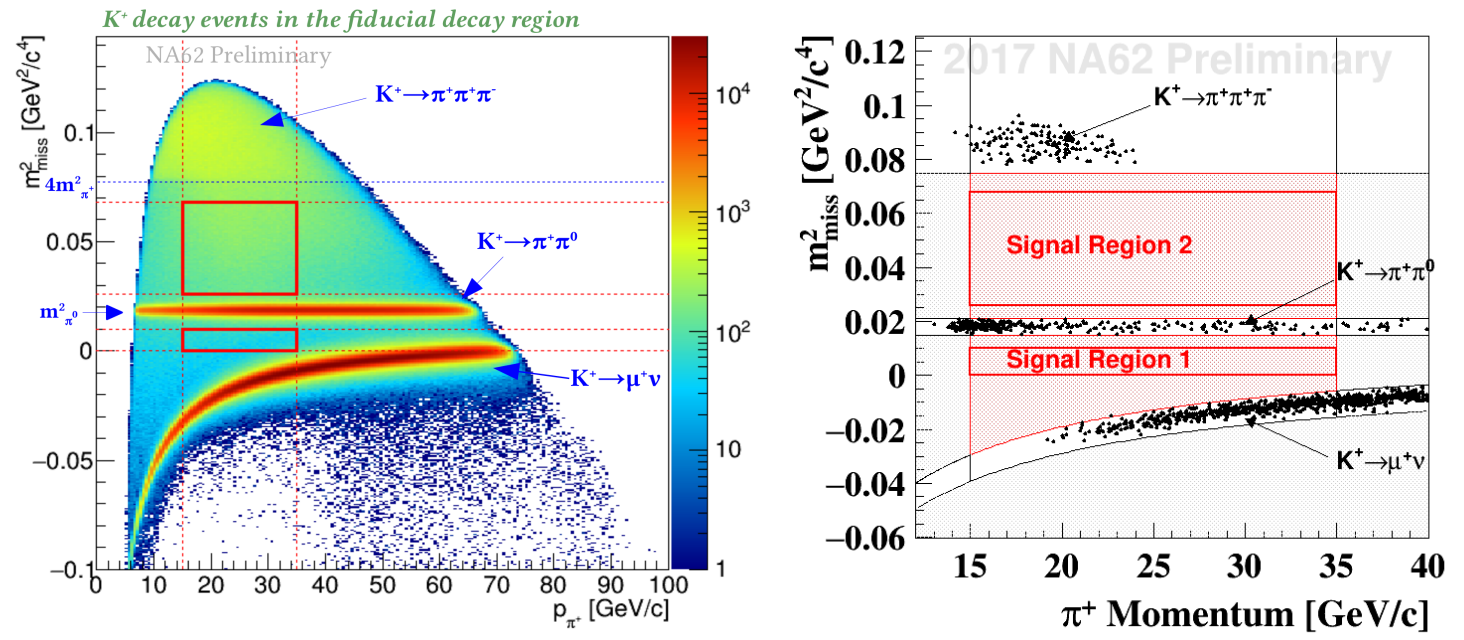

Figure 2: Left: Distribution of $m_{m i s s}^{2}$ as a function of track momentum for events selected on minimum bias data; The bands corresponding to $K^{+} \rightarrow \pi^{+} \pi^{0}$ and $K^{+} \rightarrow \mu^{+} v_{\mu}$ decays are clearly visible; the signal regions (red box) are drawn for reference. Right: Distribution of $m_{\text {miss }}^{2}$ after the complete $K^{+} \rightarrow \pi^{+} v \bar{v}$ selection. Signal and background control regions are represented by the red shaded areas. Those regions are kept blind until completion of the analysis.

Gigatracker (GTK) detector, composed of three silicon pixel stations of $6 \times 3 \mathrm{~cm}^{2}$ surface exposed to the full $750 \mathrm{MHz}$ beam rate. The GTK is used to timestamp and measure the momentum of the beam particles before entering the vacuum region downstream. The CHANTI detector, placed after the Gigatracker, tags hadronic beam-detector interactions in the last GTK station. Downstream, the magnetic spectrometer made of four straw chambers and a dipole magnet between the second and third chamber is used to measure the momentum of the charged $K^{+}$decay particles. A $17 \mathrm{~m}$ long RICH counter filled with neon gas is used to separate $\pi^{+}, \mu^{+}$and $\mathrm{e}^{+}$. The time of charged particles is measured both with the RICH and with an array of scintillators (CHOD) located downstream of the RICH. Two hadronic calorimeters (MUV1 and MUV2) and a fast scintillator array (MUV3) provide further separation between $\pi^{+}$and $\mu^{+}$. A set of photons vetoes (LAVs, LKr, IRC, SAC) hermetically cover angles up to $50 \mathrm{mrad}$ to reject extra electromagnetic activity. A detailed description of the apparatus and its performances in 2015 can be found in the NA62 beam and detector paper [ए]].

\section{The $K^{+} \rightarrow \pi^{+} v \bar{v}$ analysis}

The analysis of the complete 2017 data set is presented here, corresponding to a total number number of kaon decays $N_{K}=1.3(1) \times 10^{12}$, ten times larger than the 2016 data set. The experimental signature of the $K^{+} \rightarrow \pi^{+} v \bar{v}$ decay is the presence of a single track in the initial and final state denoted as upstream and downstream track, respectively. The main kinematic variable is $m_{\text {miss }}^{2} \equiv\left(p_{K}-p_{\pi^{+}}\right)^{2}$, where $p_{K}$ and $p_{\pi^{+}}$are the 4-momenta of the $K^{+}$and $\pi^{+}$respectively.

Events with a single-track decay topology are selected using the downstream detectors STRAW, CHOD and RICH. The track must have a matching pair of slabs in the CHOD and a reconstructed ring in the RICH, where the time is measured with 100 ps resolution. The downstream track is 
associated to an in-time kaon in the KTAG detector. The $K^{+}$track is then reconstructed and timestamped in the GTK detector. A kaon decay vertex is created at the intersection point of the GTK and STRAW tracks. The kaon decays within a $50 \mathrm{~m}$ fiducial region beginning $10 \mathrm{~m}$ downstream to the last GTK station (GTK3) are selected. The selected single-track events are shown in Figure 】, left.

The downstream track is identified as a $\pi^{+}$using two complementary methods: multivariate analysis with Boosted Decision Trees (BDT) using energy deposition, energy sharing and shower shape profiles in the electromagnetic ( $\mathrm{LKr}$ ) and hadronic calorimeters (MUV1/2), as well as signals from the muon veto (MUV3); cut-based approach using the particle mass reconstructed by the RICH detector and a track-driven likelihood discriminant for $\pi / \mu / e$ separation. The algorithm for particle identification has an efficiency for identifying $\pi^{+}$of $64 \%$ and a muon mis-identification probability of $10^{-8}$, which is at the required level for the $K^{+} \rightarrow \pi^{+} v \bar{v}$ analysis. Additionally a photon and multi-track rejection is applied based on information in all electromagnetic calorimeters (LKr, LAV, SAC and IRC) and the charged hodoscope (CHOD) providing $\pi^{0}$ suppression $\varepsilon_{\pi^{0}}=$ $(1.4 \pm 0.1) \times 10^{-8}$. The $m_{\text {miss }}^{2}$ distribution after the complete signal selection is shown in Figure $\square$, right. The selection is similar to the 2016 analysis [प]] with improvements in the reconstruction of electromagnetic energy in the $\mathrm{LKr}$ and a better pileup treatment.

The Single Event Sensitivity (S.E.S.) of the analysis of the complete 2017 data set is

$$
\text { S.E.S. }=(0.34 \pm 0.04) \times 10^{-10},
$$

which corresponds to a number of expected SM signal events $N_{\pi v \overline{\bar{v}}}^{\text {expect }}=2.5 \pm 0.4$. The signal expectation was normalized to $K^{+} \rightarrow \pi^{+} \pi^{0}$ decays selected similarly to $K^{+} \rightarrow \pi^{+} v \bar{v}$, but without photon and multi-track rejection applied. The background contamination from $K^{+}$decays is $N_{b g}=$ $0.76 \pm 0.10$. The estimate of the background from upstream decays is still on-going.

\section{Searches for LFV/LNV $K^{+} \rightarrow \pi^{-} l^{+} l^{+}$decays}

Decays of the form $K^{+} \rightarrow \pi^{-} l^{+} l^{+}$, with same-sign dielectron or dimuon pairs, violate conservation of total lepton number $L$ and lepton flavour numbers $L_{e}$ or $L_{\mu}$. The conservation of $L$ and $L_{l}(l=e, \mu, \tau)$ are emergent properties of the SM but are not explicitly required and in many BSM scenarios lepton number violating (LNV) and/or lepton flavour violating (LFV) processes are predicted. For example $K^{+} \rightarrow \pi^{-} l^{+} l^{+}$could occur mediated via Majorana neutrinos [ㅁ], [13]. Observation of LNV would be a clear indication of new physics.

The data set used for the LNV searches is a subset of the data collected by NA62 in 2017, corresponding to three months of continuous data taking. The data is collected using dedicated trigger lines for multi-track final states with $e^{ \pm}$or $\mu^{ \pm}$. The Standard Model decays $K^{+} \rightarrow \pi^{+} e^{+} e^{-}$ and $K^{+} \rightarrow \pi^{+} \mu^{+} \mu^{-}$were used as normalization for the $K^{+} \rightarrow \pi^{-} \mu^{+} \mu^{+}$and $K^{+} \rightarrow \pi^{-} e^{+} e^{+}$ analysis, respectively. The selection of signal and normalization is almost identical, differing only by the charge of the tracks in the final state. This allows important cancellations between dominant systematic uncertainties like trigger and detector efficiencies and pileup effects. 

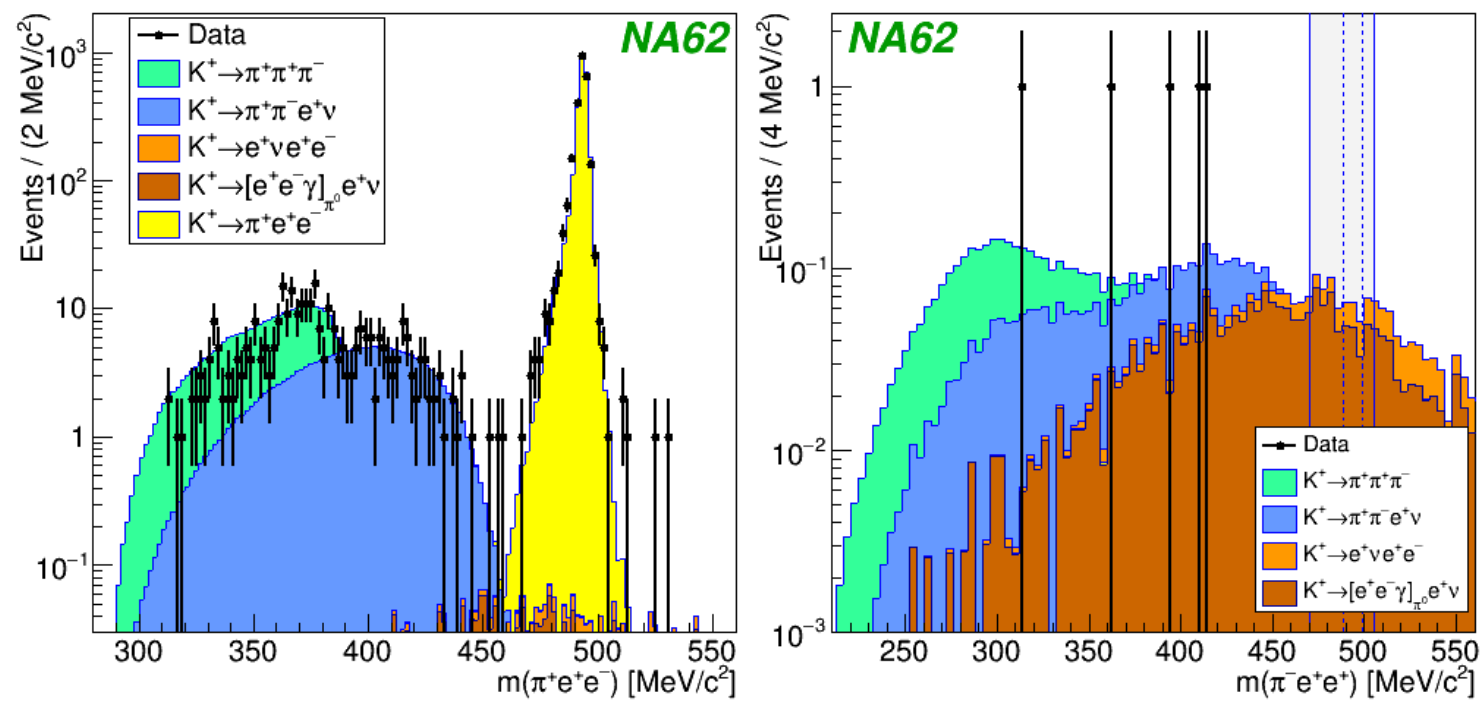

Figure 3: Left: Invariant mass spectrum for candidate SM $K^{+} \rightarrow \pi^{+} e^{+} e^{-}$events. Right: Invariant mass spectrum for candidate $\mathrm{SM} K^{+} \rightarrow \pi^{-} e^{+} e^{+}$events.

\section{1 $K^{+} \rightarrow \pi^{-} e^{+} e^{+}$result}

The $K^{+} \rightarrow \pi^{+} e^{+} e^{-}$decay mode used for normalization is selected with a common selection to the LNV mode except the charge and PID assignments. The PDG world average of the normalisation mode is $B R\left(K^{+} \rightarrow \pi^{+} e^{+} e^{-}\right)=(3.00 \pm 0.09) \times 107$ (with most recent result from NA48/2 using 7253 candidates with $M_{e e}>140 \mathrm{MeV} / c^{2}$ ). The RICH is used for positive identification of $e^{+}$, this decision was taken before unblinding and was motivated by reduction of the background in the signal region by a factor of 6 and improved discovery potential. The final $M_{\pi e e}$ invariant mass spectra for selected SM and LNV candidates is shown in Figureß3, left and Figureß], right, respectively. Precise description of the background in the lower control region in the SM is limited due to dependence of the RICH response on the event topology in a three-track environment with overlapping Cherenkov rings. This effect is accounted for with a $15 \%$ relative systematic uncertainty. For the LNV mode the total background in the signal region $(493.7 \pm 5.1) \mathrm{MeV} / c^{2}$ is $0.16 \pm 0.03$. After unblinding 0 candidate events are found in the signal region and an upper limit is established on the branching ratio of the LNV mode using the CLs statistical treatment [14]]. Using the number of kaon decays in the fiducial decay region $N_{K}=\left(2.14 \pm 0.04_{\text {stat }} \pm 0.06_{\text {ext }}\right) \times 10^{11}$ and LNV signal acceptance from uniform phase space MC simulation of $A=4.98 \%$ the single event sensitivity is S.E.S. $=(9.4 \pm 0.3) \times 10^{-11}$. An upper limit is therefore established at $B R\left(K^{+} \rightarrow \pi^{-} e^{+} e^{+}\right)<2.2 \times 10^{-10}$ at $90 \%$ confidence level.

\section{2 $K^{+} \rightarrow \pi^{-} \mu^{+} \mu^{+}$result}

The $K^{+} \rightarrow \pi^{+} \mu^{+} \mu^{-}$decay mode is used for normalization $\left(B R\left(K^{+} \rightarrow \pi^{+} \mu^{+} \mu^{-}\right)=(9.62 \pm\right.$ $0.25) \times 10^{-8}$ ). Here the RICH is not used for PID. In the SM signal region $484<M_{\pi \mu \mu}<$ $504 \mathrm{MeV} / c^{2}$ (blinded region for LNV mode) $8357 \mathrm{SM}$ candidate events are selected. The agreement between the observed and predicted number of events just outside of the signal region (background control regions) is within $3 \%$. The total background predicted in the LNV signal region 

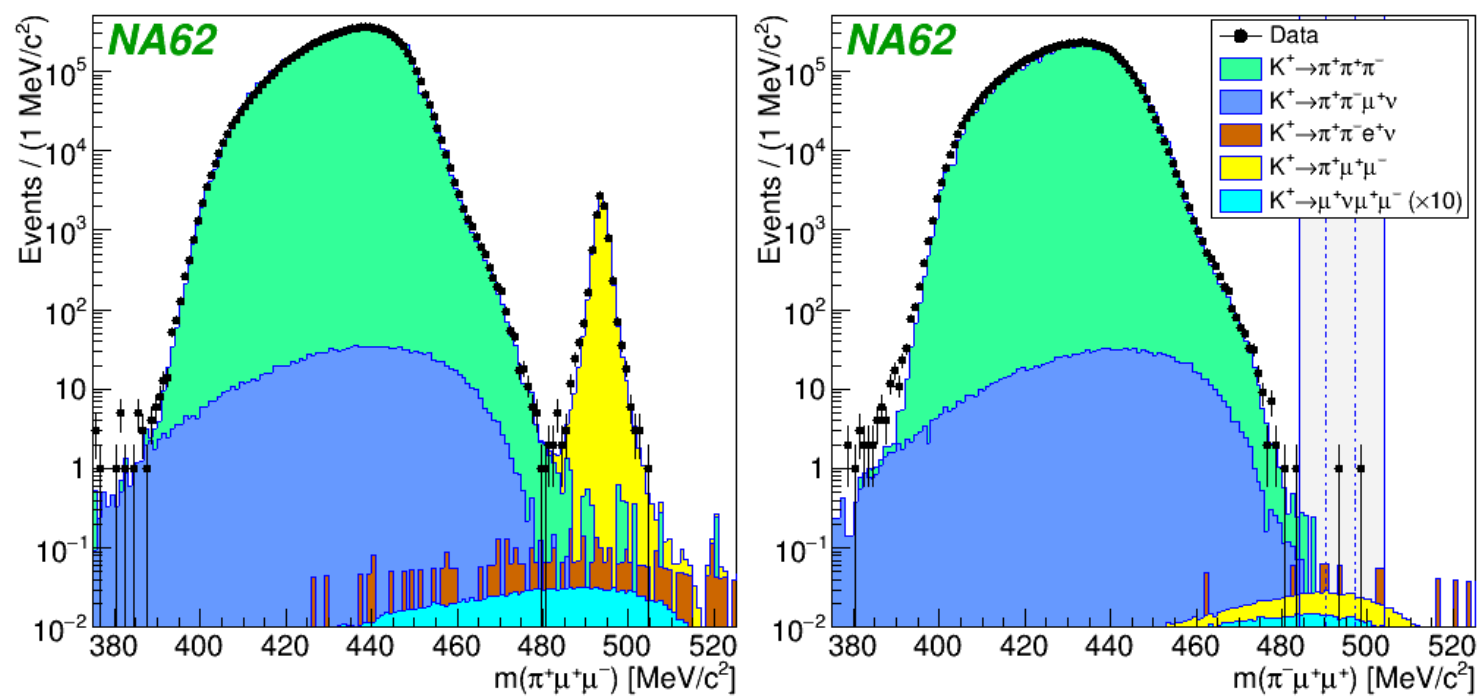

Figure 4: Left: Invariant mass spectrum for candidate SM $K^{+} \rightarrow \pi^{+} \mu^{+} \mu^{-}$events. Right: Invariant mass spectrum for candidate $\mathrm{SM} K^{+} \rightarrow \pi^{-} \mu^{+} \mu^{+}$events.

$\left(493.7 \pm 3.3 \mathrm{MeV} / c^{2}\right)$ is $0.91=p m 0.41$. The $M_{\pi \mu \mu}$ spectra for the SM and LNV modes are shown in Figure田, left and Figure田, right, respectively. After unblinding one event is found in the signal region and upper limits are set using the CLs method. Using $N_{K}=\left(7.94 \pm 0.09_{\text {stat }} \pm 0.21_{\text {ext }}\right) \times 10^{11}$ and acceptance $A=9.81 \%$ the single event sensitivity is S.E.S. $=(1.28 \pm 0.04) \times 10^{-11}$. The resulting upper limit is $B R\left(K^{+} \rightarrow \pi^{-} \mu^{+} \mu^{+}\right)<4.2 \times 10^{-11}$ at $90 \%$ confidence level.

\section{References}

[1] M. Blanke, A. J. Buras, B. Duiling, K. Gemmler , S. Gori, JHEP 903, 108 (2009)

[2] A. J. Buras, D. Buttazzo, R. Knegjens, JHEP 1511, 166 (2015)

[3] T. Blazek, P. Matak, Int. J. Mod. Phys. A 29.no.27, 1450162 (2014)

[4] Isidori et al., JHEP 0608, 064 (2006)

[5] M. Blanke, A. J. Buras, S. Recksiegel, Eur. Phys. J. C 76.no.4, 182 (2016)

[6] J. Isidori, M. Bordone, D. Buttazzo, J. Monnard, Eur. Phys. J. C 77, 618 (2017)

[7] A. J. Buras, D.Buttazzo, K.Girrbach-Noe, R. Knegjens, JHEP 11, 033 (2015)

[8] J. Brod, M. Gorbahn, E. Stammou, Phys. Rev. D 83, 034030 (2011)

[9] The E949 Collaboration, Phys. Rev. D 79, 092004 (2009)

[10] The NA62 Collaboration, JINST 12, P05025 (2017)

[11] The NA62 Collaboration, Phys. Lett. B 791, 156-166 (2019)

[12] L. Littenberg, R. Shrock, Phys. Lett. B 491, 285-290 (2000)

[13] A. Atre et al., JHEP 0905, 030 (2009)

[14] A.L. Read, J. Phys. G28, 2693 (2002) 